\title{
Inviscid mean flow through and around groups of bodies
}

\author{
By I. EAMES ${ }^{1}$, J. C. R. HUNT ${ }^{2,3}$ AND S. E. BELCHER \\ ${ }^{1}$ Departments of Mechanical Engineering and Mathematics, University College London, Gower Street, \\ London WCIE 6BT, UK \\ ${ }^{2}$ Departments of Space and Climate Physics and Geological Sciences, University College London, \\ Gower Street, London WC1E 6BT, UK \\ ${ }^{3}$ J.M. Burgers Centre, Delft University of Technology, Delft, The Netherlands \\ ${ }^{4}$ Department of Meteorology, University of Reading, Earley Gate, Reading RG6 6BB, UK
}

(Received 12 May 2003 and in revised form 15 March)

General estimates are derived for mean velocities through and around groups or arrays of fixed and moving bodies, in unbounded and bounded domains, which lie within a defined perimeter. Robust kinematic flow concepts are introduced, namely the Eulerian spatial mean velocity $\bar{u}_{E}$ in the fluid volume between the bodies, the Eulerian flow outside the group, $\boldsymbol{u}_{E}^{(0)}$, and the Lagrangian mean velocity of material surfaces or fluid particles as they pass through the group of bodies $\left(\bar{u}_{L}^{(S)}, \bar{u}_{L}^{(P)}\right)$. The Eulerian mean velocity is related to the momentum in the fluid domain, and is mainly influenced by fast moving regions of the flow. The Lagrangian mean velocity weights slowly moving regions of flow and is related to how material sheets deform as they are advected through groups of bodies. When the bodies are well-separated, the interstitial Eulerian and Lagrangian mean velocities $\left(\bar{u}_{E}^{(I)}, \bar{u}_{L}^{(I)}\right)$, are defined and calculated in terms of the far-field contributions from the velocity or displacement field within the group of bodies.

In unbounded flow past well-separated bodies situated within a rectangular perimeter, the difference between the Eulerian and Lagrangian mean velocity is negligible (as the void fraction of the bodies, $\alpha \rightarrow 0$ ). Within wide and short rectangular arrays, the Eulerian mean velocity is faster than the free-stream velocity $U$ because most of the incident flow passes through the array and $\bar{u}_{E}=U(1-\alpha)^{-1}$. Within long and thin rectangular arrays (and other cases where the reflux velocity is negligible), the Eulerian mean velocity, $\bar{u}_{E}=U\left(1-\left(1+C_{m}\right) \alpha\right) /(1-\alpha)$, is slower than the freestream velocity, because most of the incident flow passes around the array. For a spherical or circular arrays of bodies, the particle Lagrangian mean velocity is $\bar{u}_{L}^{(P)}=U\left(1+C_{m} \alpha\right)^{-1}$ and differs from $\bar{u}_{E}$. These calculations are extended to examine the mean and interstitial flow through clouds of bodies in bounded channel flows.

The new concepts are applied to calculate the mean flow and pressure between and outside clouds of bodies, the average velocity of bubbly flows as a function of void fraction, and the tendency of clouds of bubbles to be distorted depending on their shape.

\section{Introduction}

Many practical mechanical and processing engineering flows consist of collections of fixed or moving bodies. These may be unbounded or bounded high-Reynoldsnumber flows through 'clouds' of bodies, such as crop 'canopies' or buildings in the 
atmospheric boundary layer, boiler tubes in a furnace, flows through moving objects such as icebergs in the ocean, or bubble swarms in pipes. For many purposes, the main goal is to calculate various integral or statistical properties of the velocity field in relation to the distribution and movement of the bodies in order to estimate, for example, the mean drag exerted on the ambient flow, and average heat and mass transfer rates. This requires calculating the average properties of the flows through groups of bodies, which is a difficult question to answer, in part because of its ambiguity. Two contrasting approaches are used. The first, typically employed in multiphase and 'canopy' models, is to estimate the relative slip between the phases using a spatial (or phase) average of the flow field in the group of bodies (an Eulerian average; Drew \& Wallis 1992). The second approach, which is useful for some multiphase flow problems, is to define an average based on the residence time in a fluid region (a Lagrangian average). When the bodies are well-separated, it is also useful to divide these averages into two components: near- and far-field contributions to the flow or displacement field. Averaging the far-field flow (Teshukov \& Gavrilyuk 2002) or the far-field displacement field over the group of bodies yields a useful 'interstitial' mean velocity. Since the zone of validity and magnitude of these average flow quantities differ from each other, in different conditions, they need to be clearly defined and to be put on a firmer theoretical foundation. This enables important practical estimates of the flow to be calculated unambiguously, which is the aim of this paper.

As bodies translate, they displace or transport fluid in the direction in which they move. This may in turn cause a return flow or reflux. A simple example is bubbles rising in a beaker of water. The water they transport with them has to return downwards when the bubbles leave through the top surface. To analyse the flow in the interior of the beaker, the complex flows at the free surface (e.g. Eames, Hunt \& Belcher 1996) associated with the movement of the bubbles do not have to be evaluated, providing there is an appropriate definition of the mean velocity field using a Lagrangian framework. Such a Lagrangian framework is based substantially on Darwin's (1953) analysis of material surface deformation by the flow around rigid bodies. Darwin (1953) showed that provided a material surface starts infinitely far in front of the body, and is swept infinitely far past the body, the total volume displaced forward is $C_{m} V_{B}$, where $V_{B}$ is the body volume and $C_{m}$ is the added-mass coefficient. In bounded flows, a return flow or reflux is also present. The consequence of reflux is that rising bubbles (or falling particles) see, on average, a slower mean flow which leads to a hindered rise (or settling) speed.

The limitation of Darwin's analysis is that the flow must be describable by a velocity potential. Even high-Reynolds-number flow past clean bubbles generates vorticity on the bubble surface by a shear free boundary condition. However, the presence of strong irrotational straining motion in the near-wake region tends to annihilate the vorticity (Hunt \& Eames 2002). Thus even though vorticity is present locally, its effect on the displacement field tends to be weak. This explains why estimates of the drift volume based on irrotational theory are surprisingly close to experimental measurements for both three-dimensional (Bataille, Lance \& Marie 1991) and twodimensional bubbles (Bush \& Eames 1998). In the latter case, the reflux field was also measured and was in accordance with inviscid predictions based on treating the bubble and stable wake as a compound body, and the exterior flow as irrotational. Based on such irrotational flow calculations, Kowe et al. (1988) proposed a model of the interstitial flow between bubbles rising in a channel, by considering the flux transported by bubbles, but (unlike van Wijngaarden 1993) neglected the interaction 
between the bubbles. Their predictions agreed with Zuber \& Findlay's (1965) hindered rise speed formula and drift-flux relations which are widely used in multiphase flows, even for void fractions close to 0.1 (Couet, Brown \& Hunt 1991). The distinction between different contributions to the velocity field was also proposed, for bubbly flows, by Cook \& Harlow (1984), who suggested that the local flux transported by the bubbles (a near-field contribution to the velocity field) and the average flow of the gas and liquid be represented. More recently, Teshukov \& Gavrilyuk (2002) studied the average properties of oscillating bubbles in unbounded flows which involved making a distinction between the average and the far-field contribution to the velocity field. The distinction between the different contributions to the velocity field is analysed more completely here. The pressure field computed here, which determines how the distribution of bubbles changes with time, shows how rapidly large bubbles or slugs will form from distributions of smaller bubbles.

These studies can also be applied to improving models of non-uniform flows through groups of bodies or buildings and their impact on the ambient flow. Typically, estimates of the average velocity between the bodies are derived by replacing the obstacles by a distributed drag force, while ignoring inviscid blocking by the rigid bodies. Since the latter effect also forces the approach flow to go over and around them, it may explain why, if bodies are only modelled as point forces or a distribution of drag forces, it may be necessary to assume unrealistically large values of the drag coefficient in order to account for the observed slow down of flow approaching the obstacles (Belcher, Jerram \& Hunt 2003). The inviscid analysis here can be compared with the concept of inviscid inertial stresses set up by flow between the obstacles (Finnigan 2000), an approach that has not so far led to clear quantitative results.

A new framework is developed to calculate and interpret the mean flow through groups of bodies. In $\S 2$, we define the Eulerian and Lagrangian mean and interstitial velocities, and develop general expressions which are applied to potential flows (in $\S 3)$ and extended to dilute arrays in $\S 4$. These concepts are applied in $\S 5$ to examine the drift-flux relations for homogeneous bubbly flows. Concluding remarks are made in $\S 6$.

\section{Eulerian and Lagrangian mean velocities}

Figure 1(a) shows a group of bodies lying within a perimeter/surface $S$, denoted by a dashed curve, in a steady uniform approach flow, $U$. The total volume of the cloud and bodies is $V$ and $V_{b}$ respectively, so that the interstitial volume is $V-V_{b}$ and the average voidage of the bodies in the group is $\alpha=V_{b} / V$. The cross-sectional area (or width) of the streamline tube, which just passes around $V$, tends to $A_{\infty}$ far upstream of the cloud.

\subsection{Definition of Eulerian mean velocity}

The main approach for modelling multiphase flow problems has been through solving conservation equations described in terms of phase-averaged mean quantities (the twofluid approach) (e.g. Biesheuvel \& Wijngaarden 1984; Zhang \& Prosperetti 1994). The Eulerian mean velocity in a control volume $V$ (such as the volume within the perimeter $S$ of the group of bodies) is defined as the velocity $u_{x}$ (for each component) averaged over the volume occupied by the fluid (i.e. the fluid space between the bodies), 


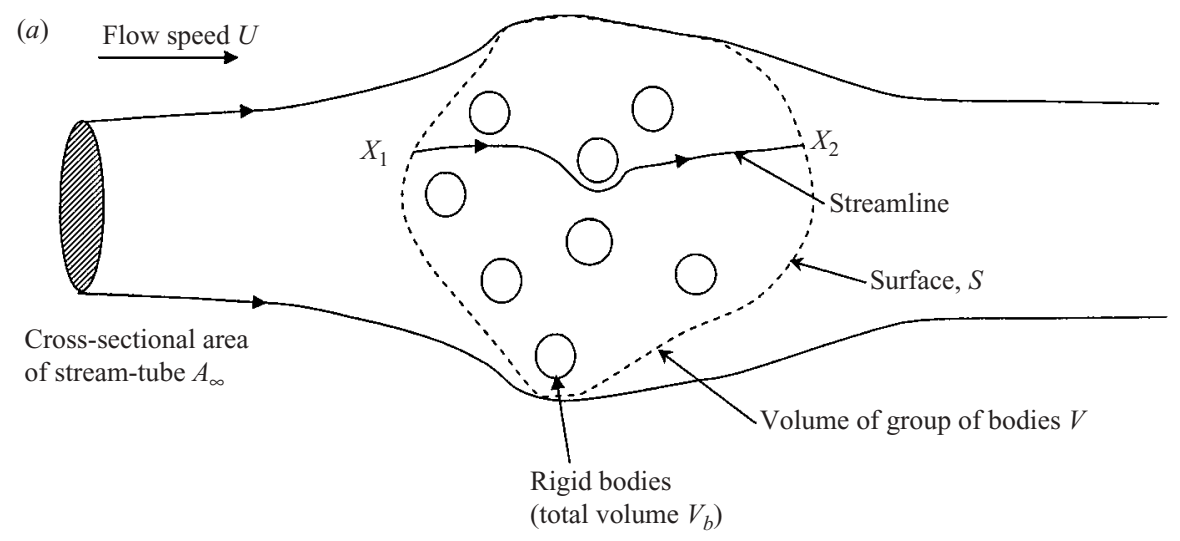

(b)

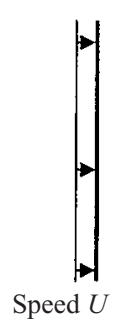

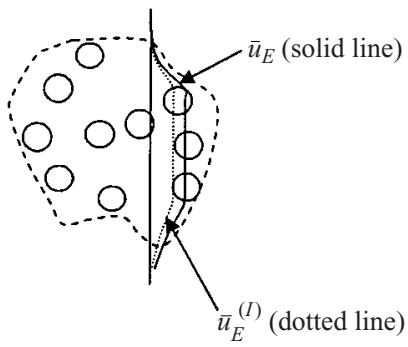

(c)

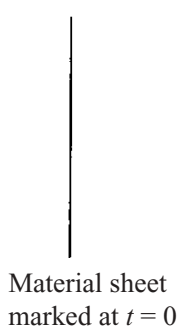

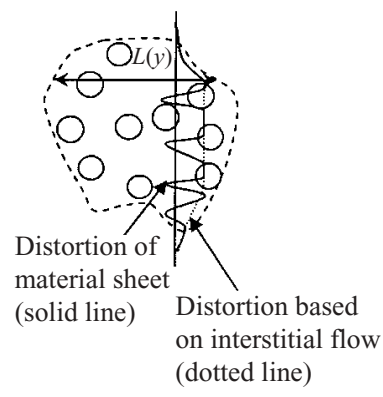

FIGURE 1. Schematic showing notation for $(a)$ the streamline flow through a group of fixed bodies, $(b)$ the Eulerian mean velocity $\bar{u}_{E}$ and interstitial velocity $\bar{u}_{E}^{(I)}$ and $(c)$ the distortions of a material surface by the bodies $(-)$ and interstitial flow $(\cdots \cdots)$. The perimeter or surface of the group of bodies, $S$, is denoted by a dashed curve.

namely

$$
\bar{u}_{E}=\frac{1}{V-V_{b}} \int_{V-V_{b}} u_{x} \mathrm{~d} V,
$$

where $V_{b}$ is the total volume of bodies in $V$ (see Wallis 1989; Drew \& Wallis 1992). The integral is taken over the fluid space between the bodies, $V-V_{b}$, and although the velocity decays slowly with distance from the individual bodies, the integral is well-defined because the volume over which the integral is taken is finite. Note that the integral may include regions of the flow with open or closed streamlines.

\subsection{Definition of Lagrangian mean velocity}

The mean Lagrangian velocity is based on the mean velocity of fluid particles advected along streamlines through $V$. Consider marked fluid particles, uniformly separated and released far upstream of the cloud of bodies. The time taken for a fluid particle to be advected from $x=x_{1}$ (point $X_{1}$ ) to some downwind position $X_{2}$ (at $x=x_{2}$ ) is $T$ (see figure $1 a$ ). The mean velocity of a fluid particle through the clouds is $L / T$, where $L=x_{2}-x_{1}$ is the horizontal distance between the two points. The travel time $T$ is related to the fluid particle displacement, $X$, (Lighthill 1956) through

$$
T=\frac{L+X}{U} \text {. }
$$




$\begin{array}{cc}\text { Notation } & \text { Description } \\ \bar{u}_{E} & \text { Eulerian mean defined } \\ & \text { over the fluid region } \\ \bar{u}_{L}^{(P)} & \text { Particle Lagrangian mean based on } \\ & \text { average of fluid particle residence time } \\ \bar{u}_{L}^{(S)} & \text { Bulk Lagrangian mean based on } \\ & \text { average speed of a material sheet } \\ \bar{u}_{E}^{(I)} & \begin{array}{c}\text { Interstitial Eulerian mean } \\ \text { between the bodies }\end{array} \\ & \begin{array}{c}\text { Interstitial Lagrangian mean } \\ \bar{u}_{L}^{(I)}\end{array} \\ & \text { between the bodies }\end{array}$

Definition

Applicable to rectangular control volumes $(2.6)$

(2.11); valid for $\alpha \ll 1$, consists of far-field contribution within the group

(2.14); valid for $\alpha \ll 1$, consists of reflux contribution

TABLE 1. Different definitions of the mean velocities. The void fraction of bodies is $\alpha$.

The displacement, $X$, is the distance a fluid particle is delayed within a flow compared to a fluid particle moving with the free-stream speed $U$ and is defined by

$$
X=\int_{0}^{T}\left(U-u_{x}\right) \mathrm{d} t
$$

Bulk estimates of the fluid particle displacement are calculated in terms of the integral of displacement across a sheet spanning the flow, $A_{\infty}$, which is defined as the partial drift volume, $D_{p}$ (see Eames, Belcher \& Hunt 1994):

$$
D_{p}=\int_{A_{\infty}} X \mathrm{~d} A \text {. }
$$

There are two (or more) possible definitions of the Lagrangian mean velocity through a region (see table 1). The 'particle' definition is the average velocity of fluid particles through the region, and is expressed as

$$
\bar{u}_{L}^{(P)}=\frac{1}{A_{\infty}} \int_{A_{\infty}} \frac{1}{T\left(y_{0}\right)} \int_{0}^{T\left(y_{0}\right)} u_{x}(x(t), t) \mathrm{d} t \mathrm{~d} A=\frac{1}{A_{\infty}} \int_{A_{\infty}} \frac{L}{T} \mathrm{~d} A .
$$

When the control volume is bounded by faces perpendicular to the mean flow (separated by a distance $L$ ), a second definition of the Lagrangian mean, based on the average speed of a material sheet (figure $1 c$ ), is

$$
\bar{u}_{L}^{(S)}=\frac{L}{\bar{T}},
$$

where the average residence time of the material sheet in the group of bodies is

$$
\bar{T}=\frac{1}{A_{\infty}} \int_{A_{\infty}} T \mathrm{~d} A .
$$

The average residence time for the material sheet is therefore related to the partial drift volume through

$$
\bar{T}=\frac{L+D_{p} / A_{\infty}}{U} .
$$

The new flow concepts defined here draw on the ideas developed by Davila \& Hunt (2001) in their analysis of the bulk settling speed of dense particles in turbulent flows.

The Lagrangian mean velocity is weighted towards regions of the flow where the residence time is largest, corresponding to stagnation points or non-slip surfaces. 
Since the mean flow is expressed in terms of the residence time along streamlines, regions of the flow surrounded by closed streamlines (such as dipolar vortices or steady wake regions) are not explicitly included in $\bar{u}_{L}^{(P)}$ or $\bar{u}_{L}^{(S)}$, but implicitly make a contribution because closed streamlines generate stagnation points, and with them a 'drift' volume. In oscillatory flows, such as those generated by progressive water waves, the mean Lagrangian velocity is often described as the mass flux or Stokes drift velocity (e.g. Stokes 1847; Ursell 1953). These key concepts have not yet been applied to multiphase or multibody problems, although they are known to be related (Eames \& McIntyre 1999).

\subsection{Interstitial flow and the effect of boundaries}

A systematic approach to characterizing the Eulerian mean velocity is developed here. First, the velocity may be decomposed into (i) a far-field flow contribution far from each body but still within the cloud of bodies - and (ii) a near-field flow contribution - local to each body. This concept, originally described qualitatively by Cook \& Harlow (1984) and Kowe et al. (1988), is strictly valid for dilute arrays since it formally requires a separation of lengthscales between the near and far field, which respectively scale as $O(a)$ and $O\left(a \alpha^{-1 / d}\right)$, where $a$ is a characteristic lengthscale of the bodies and $d$ takes the value of 2 and 3 in two- and three-dimensional flows respectively. The decomposition is defined formally here for potential flows. The farfield flow, $\boldsymbol{u}_{E}^{(f)}$, is defined mathematically to be the potential flow produced when the bodies are shrunk to zero, which yields a flow caused by the sum of the dipolar and source contributions:

$$
\nabla \cdot \boldsymbol{u}_{E}^{(f)}=\sum_{i} \Omega \boldsymbol{\mu}_{i} \cdot \nabla \delta\left(\boldsymbol{x}_{i}\right)+Q .
$$

Here $\delta\left(\boldsymbol{x}_{i}\right)$ is the delta function and $\boldsymbol{\mu}_{i}$ is the dipole moment associated with a body located at $\boldsymbol{x}_{i} ; Q$ is the source due either to the introduction of bodies, the injection of bubbles into the flow, or to satisfy kinematic boundary conditions due to the presence of walls; $\Omega$ takes the value of $2 \pi$ and $4 \pi$ in two- and three-dimensional flows respectively.

The velocity field distribution is then the combination of the uniform applied mean flow plus the response due to the distribution of sources and sinks which can be calculated by integrating the Green's function for Laplace's equation over the distribution:

$$
\boldsymbol{u}_{E}^{(f)}(\boldsymbol{x})=U \hat{\boldsymbol{x}}+\int_{V} \nabla G\left(\boldsymbol{x}, \boldsymbol{x}^{\prime}\right)\left(\nabla \cdot \boldsymbol{u}_{E}^{(f)}\left(\boldsymbol{x}^{\prime}\right)\right) \mathrm{d} \boldsymbol{x}^{\prime}
$$

(see Batchelor 1967). For planar flows, Green's function for Laplace's equation $G\left(\boldsymbol{x}, \boldsymbol{x}^{\prime}\right)=\left(\log \left|\boldsymbol{x}-\boldsymbol{x}^{\prime}\right|\right) / 2 \pi$, while for three-dimensional flows $G=-1 / 4 \pi\left|\boldsymbol{x}-\boldsymbol{x}^{\prime}\right|$. The far-field velocity distribution then determines the external flow $\boldsymbol{u}_{E}^{(0)}(\boldsymbol{x})$ outside the group of bodies. Furthermore, when they are widely separated, the bodies within the group are subjected to an interstitial flow, $\overline{\boldsymbol{u}}_{E}^{(I)}$, which is defined to be the average of $\boldsymbol{u}_{E}^{(f)}$ over the whole cloud region:

$$
\overline{\boldsymbol{u}}_{E}^{(I)}=\frac{1}{V} \int_{V} \boldsymbol{u}_{E}^{(f)} \mathrm{d} V .
$$

Thus the interstitial velocity, as described by Kowe et al. (1988), is effectively the average velocity field experienced by a test body introduced into the flow, which is not located close to any other bodies. Notice that $\overline{\boldsymbol{u}}_{E}^{(I)} \neq \overline{\boldsymbol{u}}_{E}$ because $\overline{\boldsymbol{u}}_{E}$, the Eulerian mean flow, includes contributions from the near-field flow from each body. The dipole 
(a)

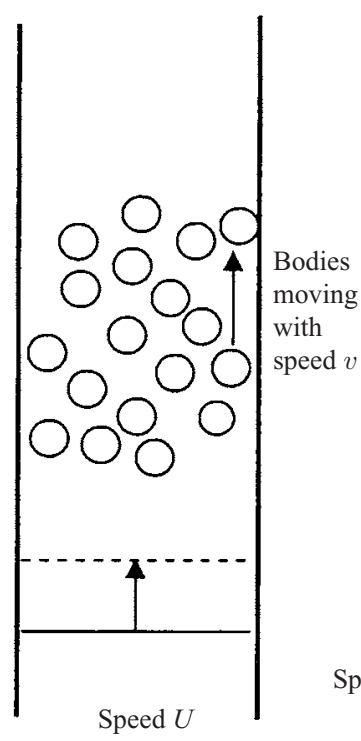

(b)

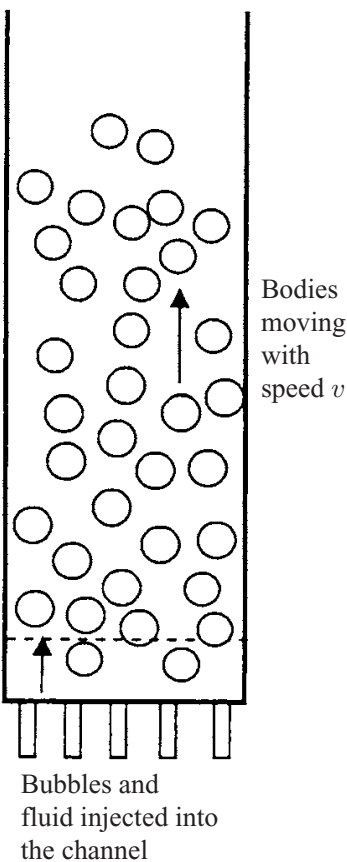

(c)

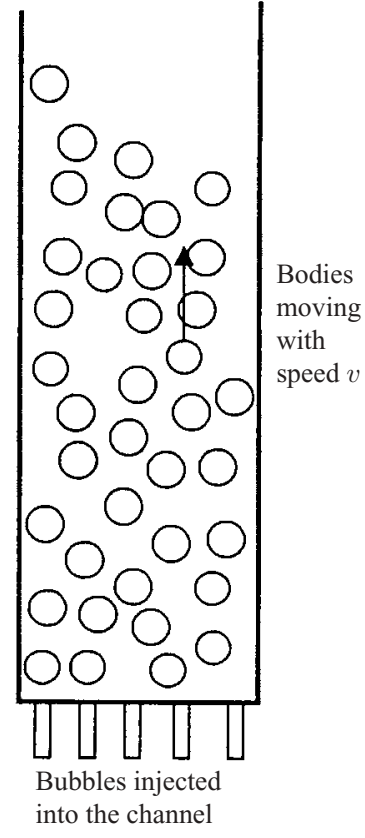

FIGURE 2. Schematics illustrating three problems with contrasting global boundary conditions. (a) A cloud of bodies spanning a channel, moving with a steady speed $v$, where the flow far upstream is uniform. $(b, c)$ The influence of a lower boundary on the kinematic and mass constraints. In $(b)$ and $(c)$, the bodies are injected into the flow - sources are required to describe the injection process and to satisfy kinematics conditions on the lower rigid wall. In $(b)$, there is a mean velocity $U$ upwind of the injection point. In $(c)$ there is no upstream flow and the Eulerian velocity is zero, but the interstitial velocity is negative.

moment associated with each body moving parallel to the stream in an interstitial Eulerian mean flow $\bar{u}_{E}^{(I)}$ is $\left(1+C_{m}\right)\left(v-\bar{u}_{E}^{(I)}\right) V_{B} / \Omega$, where $V_{B}$ is the volume of a body and $C_{m}$ its added-mass coefficient.

Teshukov \& Gavrilyuk (2002) have recently developed a statistical model of the kinetics of spherical bubbles moving in an unbounded flow, and also discuss the far-field contribution to the Eulerian velocity field. The general approach we employ in (2.9) and (2.10) reduces, for spherical bubbles, to that of Teshukov \& Gavrilyuk $(2002, \S 4)$ for unbounded flows. For unbounded flows and infinitely large groups of bodies, the integral (2.11) is not absolutely convergent and the ad hoc approach of taking its principal value was applied by Teshukov \& Gavrilyuj (2002). But, as we shall see, (2.11) may be evaluated unambiguously for bounded domains or localized clouds of bodies.

\subsection{Effect of boundaries}

Boundaries and global mass conservation impose important constraints on the interstitial velocity. Figure 2 illustrates this point with three different examples in bounded channel flows. In figure $2(a)$, the bodies are moving with a constant speed $v$. In figure 2(b) bubbles and fluid are injected into a channel, while in figure 2(c) only bubbles are injected into the channel. In examples $(b)$ and $(c)$, sources are introduced into the flow to ensure that the different kinematic boundary conditions are satisfied 
on the bottom of the tank. We first focus on bounded channel flows generated by a stream of speed $U$ through a group of bodies all moving with speed $v$ along the $x$-axis (figure $2 a$ ). When the average separation between the bodies is small relative to the separation of the channel walls, the dipole field can be replaced by a distributed dipole moment, and averaging (2.10) over the whole volume yields

$$
V \bar{u}_{E}^{(I)}+\left(1+C_{m}\right) V_{b}\left(v-\bar{u}_{E}^{(I)}\right)=V U .
$$

As a simple test we can consider groups of long and slender bodies, when $C_{m} \rightarrow 0$. The impact of the bodies is then only through blocking because the near-field flow contribution from each body is diminished and the Eulerian mean velocity is equal to the interstitial Eulerian velocity, i.e. $\bar{u}_{E}=\bar{u}_{E}^{(I)}=U /\left(1-V_{b} / V\right)$.

For the problem described by figure $2(b)$, an additional source contribution must be added to the right-hand side of (2.12) to account for the injection of the bubbles. Beyond one channel radius from the bottom of the tanks, the reflux field and interstitial mean flow are independent of distance from the lower wall, and the volumetric source added to the right-hand side of $(2.12)$ is $V_{b}\left(v-\bar{u}_{E}^{(I)}\right)$. The interstitial Eulerian mean velocity corresponding to figure $2(b)$ is then

$$
V \bar{u}_{E}^{(I)}+C_{m} V_{b}\left(v-\bar{u}_{E}^{(I)}\right)=V U
$$

The problem corresponding to figure 2(c) is also described by (2.13) with $U=0$.

As we shall show in $\S 3$, the displacement field, from which the mean Lagrangian velocity is calculated, naturally divides into a near-field (localized) drift contribution and a far-field (non-local) reflux contribution; the latter depends on the boundary condition on the whole flow. For flows through bodies moving with speed $v$, along a channel (figure $2 a$ ), the interstitial Lagrangian mean velocity is

$$
V \bar{u}_{L}^{(I)}+\left(1+C_{m}\right) V_{b}\left(v-\bar{u}_{L}^{(I)}\right)=V U,
$$

which though identical to (2.12) may be derived formally from (2.5) by identifying the interstitial component with the far-field (reflux) flow contribution to $\bar{u}_{L}^{(S)}$, as described in $\S 3$.

We have discussed different definitions of a mean velocity for steady flows through clouds of bodies. By representing bodies as dipoles, we have shown how the farfield component of the flow associated with each body (but within the array) may be estimated by averaging the far-field contribution over the entire cloud. This procedure yields the interstitial Eulerian velocity. Physically, the interstitial velocity corresponds to the average velocity seen by a body placed randomly within the cloud, but far from other bodies. Finally, we have shown that boundaries influence the interstitial velocity. In the next section, we describe how these concepts can be applied in the special case when the whole flow field is described as a potential flow to calculate general expressions that will be used to illustrate the influence of cloud shape and boundaries on the mean flow in $\S 4$.

\section{Mean potential flows through clouds of fixed bodies}

To interpret the Eulerian and Lagrangian mean velocities, we consider incompressible potential flow past a group of bodies. The flow is then equal to the gradient of a velocity potential, $\phi$, so that $\boldsymbol{u}=\nabla \phi$, and the velocity potential satisfies Laplace's equation, $\nabla^{2} \phi=0$. This broad class of flows includes macroscale Darcy flow through porous inclusions embedded in a matrix of material of uniform permeability (Eames \& Bush 1999), layerwise flow through a Hele-Shaw cell, and the flow outside the 
boundary layer and non-separating wakes of clean high-Reynolds-number spherical bubbles (Moore 1963). More recently, DNS calculations of high-Reynolds-number flows through groups of closely packed cylinders (Moulinec, Hunt \& Nieuwstadt 2003) have shown that the streamline patterns and the high-Péclet-number dispersion through closely packed cylinders are primarily determined by the potential flow blocking solutions. Excluded from the class of problems we consider are bodies with circulation and flows with singular distributions of vorticity outside the bodies.

Far upstream of the bodies, the flow is a uniform stream of speed $U$. Due to the linearity of Laplace's equation, the flow generated by $N$ bodies, labelled as $1, \ldots, k, \ldots, N$, of volume $V_{B k}$, can be decomposed as the sum of the contributions from a uniform flow and from the individual bodies,

$$
\phi=U x+\sum_{k=1}^{N} \phi_{k}
$$

where $\phi_{k}$ is the velocity potential associated with the $k$ th body. $\phi_{k}$ includes interactions between bodies, so that mathematically $\phi_{k}$ is composed of an infinite number of (image) dipoles within the $k$ th body to satisfy the boundary conditions on the surface of each body and rest of the array, or equivalently, an infinite distribution of multipoles (Saffman 1992). The bodies are fixed in a uniform flow so that the kinematic condition imposed on the surface of each body is

$$
\nabla \phi \cdot \hat{\boldsymbol{n}}=0
$$

where $\hat{\boldsymbol{n}}$ is directed out of the fluid domain. In general, the dipole moment $\boldsymbol{\mu}_{k}$ is a vector determined by the added-mass tensor, $\boldsymbol{C}_{m k}$, through $\boldsymbol{\mu}_{k}=-\left(\boldsymbol{I}+\boldsymbol{C}_{m k}\right) V_{B k} U \hat{\boldsymbol{x}} / \Omega$ (Taylor 1928). Although we focus on bodies which are symmetric about the mean flow $U \hat{\boldsymbol{x}}$, so that the component of the dipole moment parallel to the mean flow is $\mu_{k}=-\left(1+C_{m k}\right) V_{B k} U / \Omega$, where $C_{m k}=\hat{\boldsymbol{x}}^{T} \boldsymbol{C}_{m k} \hat{\boldsymbol{x}}$ is the added-mass coefficient which characterizes the shape of the body, these results may be easily extended to arbitrarily shaped bodies. The dipole moment is negative, indicating that the dipoles are pointing in the opposite direction to the mean flow. In the far field, the velocity potential associated with the $k$ th body, located at $\boldsymbol{x}_{k}$, is dominated by the dipole contribution:

$$
\phi_{k} \rightarrow \mu_{k} \frac{\partial G\left(\boldsymbol{x}, \boldsymbol{x}_{k}\right)}{\partial x} .
$$

The interaction between bodies is implicitly included through the added-mass coefficient (or dipole strength) which increases with $\alpha$ - this increase was explicitly calculated for groups of cylinders by Dalton \& Helfinstine (1971). In an unbounded flow, the outside flow $\boldsymbol{u}_{E}^{(0)}$ is characterized by a total dipole strength which is equal to the sum of the individual contributions from all the bodies, so that for $|\boldsymbol{x}| \gg\left|\boldsymbol{x}_{k}\right|$,

$$
\boldsymbol{u}_{E}^{(0)} \equiv \boldsymbol{u}_{E}^{(f)} \rightarrow \nabla\left[U x+\left(\sum_{k=1}^{N} \mu_{k}\right) \frac{\partial G(\boldsymbol{x}, \mathbf{0})}{\partial x}\right]
$$

The Eulerian mean velocity, evaluated using (3.1) and (2.1), is

$$
\bar{u}_{E}=\underbrace{\frac{U}{1-V_{b} / V}}_{\text {blocking }}[1-\underbrace{\sum_{k=1}^{N} \frac{\left(1+C_{m k}\right) V_{B k}}{V}}_{\text {near field }}+\int_{S} \frac{(\phi-U x) n_{x}}{U V} \mathrm{~d} S],
$$


where $\hat{\boldsymbol{n}}=\left(n_{x}, n_{y}, n_{z}\right)$ is a unit vector normal to the body surface and directed out of the control volume and $V_{b}=\sum_{k=1}^{N} V_{B k}$. The Eulerian mean is thus a linear operator and is formed by the sum of the contributions of each body. A distinction is made between the three terms in (3.5). The first is interpreted as a blocking contribution which causes the flow to speed up and does not depend on the shape of the bodies. The second term is a near-field contribution to the Eulerian mean arising from the impulse of the body and its volume. The third term is a far-field contribution to the Eulerian mean because the velocity potential decays sufficiently slowly that, even in the far field, boundary conditions on the whole flow domain are still important.

Unlike the Eulerian mean, the calculation of the Lagrangian mean flow is not simply the sum of the contributions from each body. For a potential flow, the displacement of a fluid particle, $X$, which is advected on a streamline can be decomposed exactly in terms of drift $\left(X_{d}\right)$ and reflux $\left(X_{r}\right)$ contributions (Eames et al. 1994),

$$
X=X_{d}+X_{r},
$$

where

$$
X_{d}=\int_{0}^{T} \frac{u^{\prime 2}}{U} \mathrm{~d} t, \quad X_{r}=-\left[\frac{\phi-U x}{U}\right]_{x_{i}}^{x_{f}} .
$$

The drift contribution, $X_{d}$, is the integral with time of the square of the velocity perturbation, $u^{\prime 2}=|\nabla \phi-U \hat{\boldsymbol{x}}|^{2}$, and $T$ is the transit time from the initial position $\boldsymbol{x}_{i}$ to the final position $\boldsymbol{x}_{f}$ which lie on surface $S$. The 'reflux' contribution, $X_{r}$, is determined only by the initial and final positions of the fluid particles along the streamlines. The displacement field naturally decomposes into a near-field drift contribution (since $u^{\prime 2}$ decays rapidly from each body) and a far-field reflux contribution (which decays slowly from each body). The reflux is zero if the planes $\boldsymbol{x}_{i}, \boldsymbol{x}_{f}$ are far from an isolated body, but is not zero for bodies moving in a confined space.

The particle Lagrangian mean is

$$
\bar{u}_{L}^{(P)}=\frac{U}{A_{\infty}} \int_{A_{\infty}} \frac{1}{1+X / L} \mathrm{~d} A .
$$

From (3.7), the drift contribution to the bulk Lagrangian mean,

$$
\int_{A_{\infty}} X_{d} \mathrm{~d} A=\int_{V-V_{b}} \frac{u^{\prime 2}}{U^{2}} \mathrm{~d} V,
$$

is proportional to the kinetic energy of the perturbation flow and related to the added-mass coefficients of the bodies. The above result is essentially Darwin's (1953) Proposition, derived by Eames et al. (1994). The bulk Lagrangian average velocity reduces to

$$
\bar{u}_{L}^{(S)}=U(1+\underbrace{\frac{1}{V} \sum_{k=1}^{N} C_{m k} V_{B k}}_{\text {near field }}-\underbrace{\frac{1}{U V} \int_{A_{\infty}}\left[\frac{\phi-U x}{U V}\right]_{x_{i}}^{x_{f}} \mathrm{~d} A}_{\text {far field }})^{-1} .
$$

The Lagrangian mean velocity is interpreted as the sum of two terms. The first term is a near-field drift contribution from each body (which tends to retard the mean flow) which is related to the added-mass of each body. The second term is a far-field contribution which tends to speed the flow up and is related to the reflux 
contribution to the partial drift volume. The interstitial Lagrangian mean velocity in (2.14) is identified as the far-field or reflux contribution to $\bar{u}_{L}^{(S)}$ in (3.10).

\section{Evaluation of the mean flow for potential flows through a dilute cloud of fixed bodies}

For simplicity, in the ensuing calculations, the bodies are assumed to be identical (with volume $V_{B}$ and added-mass coefficient $C_{m}$ ), though this can easily be extended. When $\alpha \ll 1$, the flow interaction between the bodies is weak and the dipole moment associated with each body tends to that generated by an isolated body in an inbounded flow, $\mu_{k}=-\left(1+C_{m}\right) V_{B} U / \Omega$.

From (3.5) and (3.10) we see that for the dilute clouds of bodies $(\alpha \ll 1)$, the local contributions to the Eulerian and Lagrangian mean velocity are identical and can be respectively interpreted as an impulse or added-mass contribution (which are identical for steady flows). However, Eulerian and Lagrangian averaging deal with the far-field contributions in an altogether different manner and can be respectively interpreted as an impulse (or volume flux) and reflux. As we shall demonstrate, in unbounded flows, these two different averaging approaches may yield altogether different results.

\subsection{Unbounded flows: planar rectangular group of obstacles}

For the special case of a planar rectangular array of planar obstacles, randomly positioned in a region $|x| \leqslant l,|y| \leqslant w$, where the rectangular region $V$ has length $2 l$ and width $2 w$, the starting and finishing positions of the material sheets are perpendicular to the undisturbed mean flow.

From (3.10) and (3.5), the Lagrangian and Eulerian mean velocities are:

$$
\bar{u}_{L}^{(S)}=U\left(1+C_{m} \alpha-\frac{1}{V} \int_{S} \sum_{k=1}^{N} \frac{\phi_{k} n_{x}}{U} \mathrm{~d} S\right)^{-1},
$$

and

$$
\bar{u}_{E}=\frac{U}{1-\alpha}\left(1-\left(1+C_{m}\right) \alpha+\frac{1}{V} \int_{S} \sum_{k=1}^{N} \frac{\phi_{k} n_{x}}{U} \mathrm{~d} S\right) .
$$

The bulk Lagrangian and Eulerian mean velocities (according to (4.1) and (4.2)) are consistent to $O(\alpha)$ because the far-field contributions to the average velocity are dealt with in an identical manner. Also, by expanding the integrand in (3.8), the particle Lagrangian mean velocity can be seen to be consistent to $O(\alpha)$ with $\bar{u}_{L}^{(S)}$. Thus, in this example, the difference between the Lagrangian and Eulerian mean velocities is negligible for $\alpha \ll 1$.

The reflux contribution from a body (represented as a dipole) located at $\left(x_{k}, y_{k}\right)$, corresponding to the third terms in (4.1) and (4.2), is evaluated using (3.3), to give

$$
\begin{aligned}
\int_{S} \frac{\phi_{k} n_{x}}{U} \mathrm{~d} y=-\frac{\left(1+C_{m}\right) V_{B}}{2 \pi}[ & \tan ^{-1}\left(\frac{w-y_{k}}{l-x_{k}}\right)+\tan ^{-1}\left(\frac{w+y_{k}}{l-x_{k}}\right) \\
& \left.+\tan ^{-1}\left(\frac{w-y_{k}}{l+x_{k}}\right)+\tan ^{-1}\left(\frac{w+y_{k}}{l+x_{k}}\right)\right] .
\end{aligned}
$$

When the separation of the bodies is much smaller than the size of the rectangular array (i.e. $a \alpha^{-1 / 2} \ll l, w$ ), the Eulerian mean velocity may be evaluated by 
averaging (4.3) over the rectangular array to yield

$$
\begin{aligned}
\bar{u}_{E}= & \frac{U}{1-\alpha}\left[1-\left(1+C_{m}\right) \alpha\right. \\
& \left.+\frac{2\left(1+C_{m}\right) \alpha}{\pi}\left[\tan ^{-1}(w / l)-\frac{l}{4 w} \log \left(1+w^{2} / l^{2}\right)+\frac{w}{4 l} \log \left(1+l^{2} / w^{2}\right)\right]\right] .
\end{aligned}
$$

For short wide arrays of obstacles $(l / w \ll 1)$, the mean Eulerian velocity (from (4.4)) tends to $U(1-\alpha)^{-1}$ and is faster than $U$ due to a blocking effect which accelerates the flow through the obstacles. As the aspect ratio of the rectangular region $(l / w)$ increases, the mean Eulerian velocity (from (4.4)) tends to $U\left(1-\left(1+C_{m}\right) \alpha\right) /(1-\alpha) \approx$ $U\left(1-\alpha C_{m}\right)$ and is slower than $U$ because the residence time is increased by the stagnation regions. Thus there are two important contributions to the average velocity: a blocking effect which speeds up the flow and a local added-mass or drift effect which retards the flow near the stagnation points.

Given the different effects of extreme cloud shapes on $\bar{u}_{L}^{(S)}, \bar{u}_{L}^{(P)}$ and $\bar{u}_{E}$, it is interesting to study an intermediate shape, typical of a rising cloud of bubbles.

\subsection{Unbounded flows: circular/spherical group of bodies}

In the previous example, the difference between the Eulerian and Lagrangian mean velocities was small. We elaborate here on an important example of flow through a circular or spherical cloud (of radius $w$ ) of bodies where there are greater differences between these mean quantities.

From the expression for the Eulerian mean velocity for potential flows given by (3.5) and in the Appendix, the Eulerian mean velocity, within a circular or spherical cloud of bodies, is

$$
\bar{u}_{E}=U\left(\frac{1-\left(1+C_{m}\right) \alpha+(1 / d)\left(1+C_{m}\right) \alpha}{1-\alpha}\right),
$$

where $d$ takes the value of 2 and 3 for two- and three-dimensional flows respectively. Each body leads to a flux of fluid $\left(1+C_{m}\right) V_{B} U / d$ out of the circular or spherical control volume $V$, which tends to speed up the interior flow. For the special case of a circular/spherical cloud of cylinders/spheres, characterized by $C_{m}=1, \frac{1}{2}$ respectively, the Eulerian mean in the fluid space is exactly $U$, as also recognised by Jankovic, Fiori \& Dagan (2003).

For $\alpha \ll 1, \mathrm{~d} A / L=n_{x} \mathrm{~d} S / L \approx \mathrm{d} S / w$. The up- and downstream surfaces of the control volume are not planes and the definition of the bulk Lagrangian mean is not applicable. The far-field or reflux contribution to the particle Lagrangian mean velocity is zero:

$$
\int_{A} \frac{X_{r}}{L} \mathrm{~d} A=\sum_{k=1}^{N} \frac{1}{U w} \int_{S} \phi_{k} \mathrm{~d} S=0
$$

(see the Appendix). Thus the average reflux contribution to the displacement of a fluid particle (or its effect on the mean travel time) is zero, independent of the position of the bodies. Combined with the drift contribution, the particle Lagrangian mean velocity in a circular or spherical array is

$$
\bar{u}_{L}^{(P)}=U\left(1-C_{m} \alpha\right),
$$

slightly slower than the ambient flow. This example serves to illustrate a case when the Eulerian and Lagrangian mean velocities are different, with $\bar{u}_{E}$ larger than $\bar{u}_{L}$ by a factor $\left(1+C_{m}\right) \alpha / d$. 


\subsection{Bounded channel flows}

Bounding channel walls have a significant effect on the far-field contribution to the Eulerian and Lagrangian mean velocities. We consider the flow through a fixed array of bodies of length $L$, which is much longer than the channel width $W$. In this limit the final results are independent of $L$ and $W$.

By mass conservation, the integral of fluid displacement across the channel caused by a material surface, marked upstream of the array and being advected through the array, is

$$
\frac{1}{A_{\infty}} \int_{A_{\infty}} X \mathrm{~d} A=-\alpha L
$$

provided there are no closed streamlines, where $V=A_{\infty} L$. Thus the bulk Lagrangian mean velocity is

$$
\bar{u}_{L}^{(S)}=U(1-\alpha)^{-1} \text {. }
$$

The drop in the velocity potential across a long array (for $L \gg W$ ) is

$$
\int_{A_{\infty}}\left(\phi-U_{x}\right) n_{x} \mathrm{~d} S=\left(1+C_{m}\right) \alpha L A_{\infty} U,
$$

so that the Eulerian mean velocity is

$$
\bar{u}_{E}=U(1-\alpha)^{-1} \text {. }
$$

Thus for flow bounded by channel walls, the Eulerian and bulk Lagrangian mean velocities are identical, $\bar{u}_{L}^{(S)} \equiv \bar{u}_{E}$, and faster than the flow far upstream of the bodies as a consequence of flow blocking by obstacles, and independent of the shape of the bodies.

From (2.12) and (2.14), the interstitial Eulerian and Lagrangian mean velocities are

$$
\bar{u}_{E}^{(I)}=\bar{u}_{L}^{(I)}=U\left(1-\left(1+C_{m}\right) \alpha\right)^{-1} .
$$

The interstitial Lagrangian mean velocity may also be identified from the far-field reflux contribution in the averaging process, described in (3.10).

\section{Interstitial velocity and drift-flux relations for bubbly flows}

Most one-dimensional models of multiphase flows are built around a drift-flux description, where the difference between the volumetric flux of gas and liquid, $j_{12}$, is modelled empirically (see Wallis 1969, p. 13). As noted by Kowe et al. (1983), such expressions for the difference between these fluxes can be understood by considering how bubbles move relative to an interstitial flow. We develop these ideas for the problem described in figure 2(a), to determine the relation between the bulk gas and liquid flows. This provides a theoretical justification for Zuber \& Findlay's (1965) drift-flux correlation for medium-sized high-Reynolds-number bubbles.

The interstitial Lagrangian mean velocity corresponding to the problem described in figure 2(a), may be calculated directly from (3.10), so that

$$
\bar{u}_{L}^{(I)}=v+\frac{U-v}{1-\alpha\left(1+C_{m}\right)},
$$

which on rearrangement yields

$$
\bar{u}_{L}^{(I)}+\left(v-\bar{u}_{L}^{(I)}\right) \alpha\left(1+C_{m}\right)=U,
$$

as defined in $\S 2$. Physically, the bubbles transport both the drift volume $\left(\alpha C_{m}\right)$ and volume $(\alpha)$ with them. The drift volume has been measured experimentally and 
confirms that (5.3) provides a leading-order description of the interstitial Lagrangian flow. For low void fractions, the difference between $\bar{u}_{E}^{(I)}$ and $\bar{u}_{L}^{(I)}$ is second order. When the bubble response time is short compared to the advective timescale of the flow, the bubbles rise with terminal rise speed relative to the interstitial flow, corresponding to the average fluid speed seen by a test bubble,

$$
v-\bar{u}_{E}^{(I)}=v_{T},
$$

which implies a slip velocity

$$
v-U=v_{T}\left(1-\left(1+C_{m}\right) \alpha\right) .
$$

Empirical correlations (e.g. Govier \& Aziz 1982, p. 383) suggest that the slip velocity of the bubble is

$$
v-U=v_{T}(1-\alpha)^{1.5}
$$

which is consistent to $O(\alpha)$ with (5.5) for $C_{m} \sim 0.5$, a value typical of near spherical bubbles. Van Wijngaarden (1993) calculated the average rise speed of bubbles by a detailed consideration of the pairwise interactions between bubbles in an unbounded domain, and obtained $v=v_{T}(1-1.47 \alpha)$ for bubbles rising in a stagnant ambient fluid $(U=0)$. As we have shown, the difficulty of considering unbounded flows, which is related to the divergence of the mean Eulerian velocity, is overcome by considering bounded channel flows. Then the mean bubble slip velocity is independent of the pairwise distribution of bubbles provided they are well-separated.

For homogeneous bubbly flows, the drift-flux, $j_{12}$, defined in terms of the difference of the slip velocities between the gas and liquid phase, is

$$
j_{12} \equiv \alpha(1-\alpha)(v-U)
$$

which when combined with (5.5) yields

$$
j_{12}=v_{T} \alpha(1-\alpha)\left(1-\left(1+C_{m}\right) \alpha\right) .
$$

Empirical correlations for drift-flux are based on fitting the exponent $n$ from the expression

$$
j_{12}=v_{T} \alpha(1-\alpha)^{n}
$$

to experimental data. The value of $n$ depends on the particular configuation considered, but a value of $n=2.5$ is recommended for high-Reynolds-number homogeneous bubbly flows, which is consistent to $O\left(\alpha^{2}\right)$ with (5.8) for near spherical bubbles where $C_{m} \sim 0.5$. The conclusion from these calculations is that the concept of an interstitial Eulerian mean velocity provides a bulk description of the impact of multiple bubbles on the mean flow.

The pressure field averaged over a region between the bubbles is

$$
\bar{p}_{E}=\frac{1}{V-V_{b}} \int_{V-V_{b}} p \mathrm{~d} V .
$$

Coupled with Bernoulli's equation, $p=p_{0}+\frac{1}{2} \rho\left(U^{2}-u^{2}\right)$, where $p_{0}$ is the pressure far upstream, the average pressure within a cloud of bodies or bubbles rising in a channel flow is

$$
\bar{p}_{E}=p_{0}-\frac{\rho \alpha C_{m}(U-v)^{2}}{2(1-\alpha)}
$$

(e.g. see Kowe et al. 1988) so that the average pressure in a homogeneous distribution of bubbles rising in a channel is reduced. This is why in a layer of bubbles rising in a 
(a)

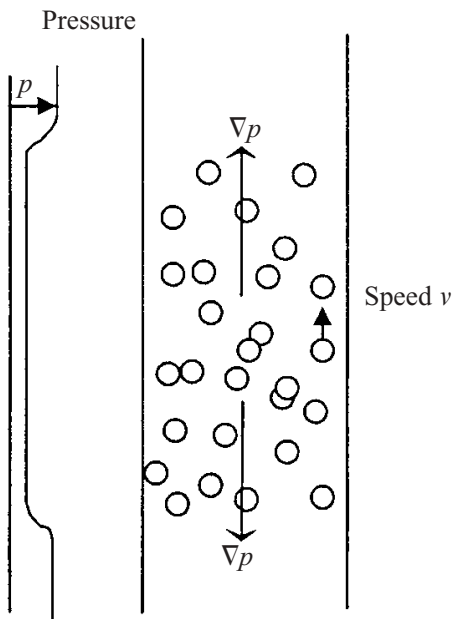

(b)

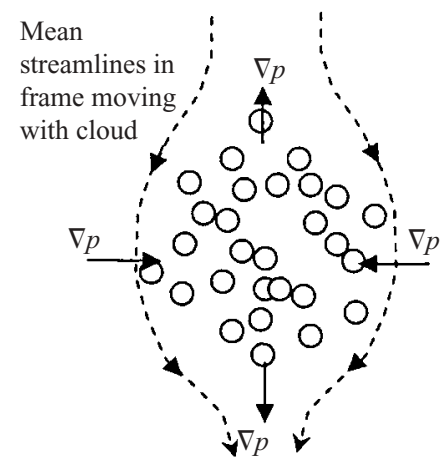

Pressure,

FiguRE 3. A schematic of the pressure field in $(a)$ bounded homogeneous bubbly flow where bubbles are rising with speed $v$ in a channel and $(b)$ a cloud of bubbles rising in an unbounded channel. In $(a)$, the mean flow speeds up through the array of bubbles, leading to a reduction of pressure. The direction of the pressure gradients indicates that the bubbles will tend to coalesce. In an unbounded flow, $(b)$, the acceleration of the flow around the cloud means that while there are vertical pressure gradients which causes the vertical height of the cloud to shrink, lateral pressure gradients cause the cloud to widen reducing coalescence and causing the cloud to grow.

pipe, those at the top and bottom will tend to converge towards the middle, causing collisions and larger bubbles to be formed (figure $3 a$ ). When the bodies or bubbles are placed in a group with finite volume $V$ far from any confining walls (e.g. a bubble cloud moving with speed $U$ ), the external velocity $\boldsymbol{u}_{E}^{(0)}$ around the volume gives rise to an additional higher pressure at the top and bottom of the cloud and lower pressure at the sides, which tends to inhibit coalescence (figure $3 b$ ).

Boundaries have a significant influence on the interstitial velocity, as described in $\S 2$ and shown in figure $2(b, c)$. When the flow is bounded by a porous or rigid lower wall, the interstitial Eulerian velocity is increased because bubbles are injected into the channel, and as a result the average rise speed is increased, according to

$$
v-U=v_{T}\left(1-C_{m}\right),
$$

faster than the example shown in figure $2(a)$.

\section{Concluding remarks}

In this paper we have worked out a framework for analysing and understanding inviscid flows through groups of bodies based on definitions of Eulerian and Lagrangian mean velocities and their decompositions into near and far (or interstitial) components. This provides a useful conceptual framework to solve certain multibody and multiphase problems.

We have shown how the definitions and magnitudes of the Eulerian and Lagrangian mean velocities differ, corresponding as they do to a phase-averaged velocity and average residence times. Both contain near-field, far-field and array-scale blocking 
contributions. The definitions of the Lagrangian and Eulerian mean properties contain integrals which require careful evaluation because they are related to the momentum of the flow and therefore yield potentially non-absolutely convergent integrals in unbounded flows. These concepts have been illustrated by studying potential flows, which include a broad class of problems, including the high-Reynolds-number flows past clean bubbles, flows through pipe bundles (where vorticity annhilation by straining motion cancels out most of the shed vorticity and where the flow is dominated by irrotational blocking by the pipes), and macroscale flows in porous media where uniform-permeability inclusions are embedded in a matrix of uniform permeability.

Though the framework has been applied to relatively simply geometries, our analysis highlights a number of fundamentally important processes. For dilute rectangular arrays, the difference between Eulerian and Lagrangian mean velocity is negligible. When the rectangular array is short and wide, the average volumetric flux (of the bodies and fluid) is constant along the mean streamlines, so that the interstitial velocity is determined by the reduced space between the bodies (i.e. blocking). This leads to an increase in the average flow. When the rectangular array is long and thin, the average flow in the cloud tends to be retarded by the local flow deceleration (drift or impulse), near the bodies. Thus different physical processes are important in different regions of an array, with blocking dominating the deceleration of the flow at the front of the array, while drift or drag retards the flow downstream (Belcher et al. 2003). For circular and spherical groups of bodies, the Eulerian and Lagrangian mean velocities differ in their weightings of the non-local contributions to the averages.

For bounded channel flows, the Eulerian and bulk Lagrangian velocities are identical (for all volume fractions) and are faster than the flow far upstream due to blocking. The most important contribution to this analysis is the concept of an interstitial Eulerian and Lagrangian mean flow, where far-field contributions to the velocity and displacement field are considered. This is in the same spirit as Kowe et al. (1983), but has been put on a firmer theoretical foundation. One important conclusion is that a weak vortical flow has a negligible impact on the interstitial Lagrangian mean because the local contribution to the drift is dominated by the local potential flow around the bubble and wake. This has been confirmed experimentally by Bataille, Lance \& Marie (1991) and Bush \& Eames (1998). These calculations justify to some extent semi-empirical relations for drift-flux which are commonly assumed in high-Reynolds-number homogeneous flows.

The calculations in this paper raise a fundamental question: if the averaging process are different, which velocity should be used? The answer lies in the type of problem considered. For problems where the residence times are important, such as reactions or the decay of chemical species, the Lagrangian mean velocity is the most relevant. But, for problems where the bodies are fixed and do not move relative to the mean interstitial flow, the Eulerian average flow is the most suitable.

The clear deficiency of the analysis presented is the neglect of the effects of vorticity shed from rigid bodies on both the mean flow and the displacement field. For closely packed bodies, the influence of shed vorticity is weak because of wake interaction and the straining flow around individual bodies which both lead to vorticity annihilation, as demonstrated by the DNS calculations of Moulinec et al. (2003). The analysis presented here gives a practical bulk estimate of the average flow in such cases. In general the influence of such bodies may be included by introducing distributed or point forces into the momentum equation. The significant and 
broad-range implications of inertial blocking and shed vorticity will form the focus of a future study.

A further challenge is to investigate whether the concept of an interstitial flow and its estimate for low void fractions is generally valid at moderate void fractions $(\sim 0.1)$, when the bubbles are not widely separated. To measure the interstitial velocity directly requires the development of new diagnostic tools to process incomplete and conditioned data sets - some of these significant challenges are currently being met by researchers at IMFT, Toulouse.

I.E. and J.C.R.H. gratefully acknowledge, the support at University College London from respectively, EPSRC at the Department of Mechanical Engineering and NERC at the Centre for Polar Observation and Modelling. We acknowledge the considerable benefit from close interactions with researchers at IMFT, in particular from discussions with Dr Veronique Roig, Dr Frederic Risso and Dr Jacques Magnaudet, for their generous hospitality and time, and also access to their preliminary experimental measurements of interstitial flow in homogeneous highReynolds-number bubbly flows.

\section{Appendix}

We demonstrate the following relationships for a dipole of strength $\mu_{i}$ located within a circular or spherical control volume (of radius $w$ ) bounded by surface $S$ :

$$
\int_{S} \phi_{i} \mathrm{~d} S=0, \quad \int_{S} \phi_{i} n_{x} \mathrm{~d} S=-\frac{\Omega \mu_{i}}{d} .
$$

The proof of (A1a) is given by Lighthill (1986, p. 123), and it is exact for a dipole in two and three dimensions. For two-dimensional flows, (A1b) may be demonstrated analytically by writing $\phi_{i}$ in polar coordinates $(r, \theta)$. On the surface $S(r=w)$,

$$
\phi_{i}=-\frac{\mu_{i}\left(r \cos \theta-r_{p} \cos \theta_{p}\right)}{r^{2}+r_{p}^{2}-2 r r_{p} \cos \left(\theta-\theta_{p}\right)}
$$

where the dipole is located at $\left(r_{p}, \theta_{p}\right)$. Substituting into (A1b) and subsequent integration yields the required results.

For three-dimensional flows, we need to consider the flux through a conic surface element of $S$, to give

$$
\int_{S} \phi_{i} n_{x} \mathrm{~d} S=\int_{S}-\frac{\mu_{i} \cos \theta}{r^{2}} \mathrm{~d} S=-\mu_{i} \int_{S} \cos ^{2} \theta \mathrm{d} \Omega .
$$

The average value of $\cos ^{2} \theta$ over the total solid angle $\Omega=4 \pi$. This value is independent of $r$ and the location of the body. Defining a unit radius sphere around the body and denoting $\mathrm{d} \Omega=\sin \theta \mathrm{d} \theta \mathrm{d} \varphi$, then

$$
\int_{S} \cos ^{2} \theta \mathrm{d} \Omega=\int_{0}^{2 \pi} \int_{0}^{\pi} \cos ^{2} \theta \sin \theta \mathrm{d} \theta \mathrm{d} \varphi=\frac{4 \pi}{3}=\frac{\Omega}{d} .
$$

Combining (A3) with (A2), yields (A1b) for three-dimensional flows. The above results have also been confirmed by numerical integration of the surface integrals. 


\section{REFERENCES}

Batchelor, G. K. 1967 An Introduction to Fluid Dynamics. Cambridge University Press.

Bataille, J., Lance, M. \& Marie, J. L. 1991 Some aspects of the modelling of bubbly flows. In Phase-Interface Phenomena in Multiphase Flows (ed. G. F. Hewitt, F. Mayinger \& J. R. Riznic), pp. 179-193. Hemisphere.

Belcher, S. E., Jerram, N. \& Hunt, J. C. R. 2003 Adjustment of a turbulent boundary layer to a canopy of roughness elements. J. Fluid Mech. 488, 369-398.

Biesheuvel, A. \& van WijngaArden, L. 1984 Two-phase flow equations for a dilute dispersion of gas bubbles in liquid. J. Fluid Mech. 148, 301-318.

Bush, J. W. M. \& Eames, I. 1998 Fluid displacement by bubbles rising in a thin gap. Intl J. Multiphase Flow 24, 411-430.

CoOK, T. L. \& Harlow, F. H. 1984 Virtual mass in multiphase flow. Intl J. Multiphase Flow 10, 691-696.

Coute, B., Brown, P. \& Hunt, A. 1991 Two-phase bubbly-droplet flow through a contraction: experiments and a unified model. Intl J. Multiphase Flow 17, 291-307.

Dalton, C. \& Helfinstine, R. A. 1971 Potential flow past a group of circular cylinders. Trans. ASME: J. Basic Engng 93, 636-642.

Darwin, C. G. 1953 A note on hydrodynamics. Proc. Camb. Phil. Soc. 49, 342-354.

Davila, J. \& Hunt, J. C. R. 2001 Settling of small particles near vortices and in turbulence. J. Fluid Mech. 440, 117-145.

Drew, D. A. \& Wallis, G. B. 1992 Fundamentals of two-phase flow modelling. Third Intl Workshop on Two-Phase Flow Fundamentals (ed. G. F. Hewitt \& R. T. Lahey).

Eames, I., Belcher, S. E. \& Hunt, J. C. R. 1994 Drift, partial drift, and Darwin's proposition. J. Fluid Mech. 275, 201-223.

Eames, I., Hunt, J. C. R. \& Belcher, S. E. 1996 Inviscid displacement by a sphere moving away from a wall. J. Fluid Mech. 324, 333-353.

Eames, I. \& Bush, J. W. M. 1999 Longitudinal dispersion by bodies fixed in a potential flow. Proc. R. Soc. Lond. A 455, 3665-3686.

Eames, I. \& McIntyre, M. E. 1999 On the connection between Stokes and Darwin drift. Camb. Phil. Soc. Proc. 126, 171-174.

Finnigan, J. J. 2000 Turbulence in plant canopies. Annu. Rev. Fluid Mech. 32, 519-571.

Govier, G. W. \& Aziz, K. 1982 The Flow of Complex Mixtures in Pipes. Krieger, New York.

Hunt, J. C. R. \& EAmes, I. 2002 The disappearance of laminar and turbulent wakes in complex and straining flows. J. Fluid Mech. 457, 111-132.

Jankovic, I., Fiori, A. \& Dagan, G. 2003 Effective conductivity of an isotropic heterogeneous medium of lognormal conductivity distribution. SIAM Multiscale Modelling, Analysis \& Simulation 1, 40-56.

Kowe, R., Hunt, J. C. R., Hunt, A., Couet, B. \& Bradbury, L. J. S. 1988 The effects of bubbles on the volume fluxes and the pressure gradients in unsteady and non-uniform flow of liquids. Intl J. Multiphase Flow 14, 587-606.

Lighthill, M. J. 1956 Drift J. Fluid Mech. 1, 31-53.

Lighthill, M. J. 1986 An Informal Introduction to Theoretical Fluid Mechanics. Oxford University Press.

Magnaudet, J. \& Eames, I. 2000 Motion of high Reynolds number bubbles in inhomogeneous flows. Annu. Rev. Fluid Mech. 32, 659-708.

Moore, D. W. 1963 The velocity of rise of distorted gas bubbles in a liquid of small viscosity. J. Fluid Mech. 16, 659-708.

Moulinec, C., Hunt, J. C. R. \& Nieuwstadt, F. T. M. 2003 Disappearing wakes and dispersion in tube bundles. J. Flow Turbulence Combust. (Submitted)

Saffman, P. G. 1992 Vortex Dynamics. Cambridge University Press.

Stokes, G. G. 1847 On the theory of oscillating waves. Trans. Camb. Phil. Soc. 8, 441-455.

TAYLOR, G. I. 1928 The energy of a body moving in an infinite fluid with applications to airships. Proc. R. Soc. Lond. A 70, 13-21.

Teshukov, V. M. \& Gavrilyuk, S. L. 2002 Kinetic model for the motion of compressible bubbles in a perfect fluid. Eur. J. Mech. B/Fluids 21, 469-491.

Ursell, F. 1953 Mass transport in gravity waves. Proc. Camb. Phil. Soc. 40, 145-150. 
Wallis, G. B. 1969 One-dimensional 2-Phase Flow. McGraw-Hill.

Wallis, G. B. 1989 Inertial coupling in two-phase flows. Multiphase Sci. Tech. 5, 239-361.

VAN WiJngaARDEN, L. 1993 The mean rise velocity of pairwise-interacting bubbles in liquid. J. Fluid Mech. 251, 55-78.

YIH, C. S. 1985 New derivations of Darwin Theorem. J. Fluid Mech. 152, 163-172.

Zhang, D. Z. \& Prosperetti, A. 1994 Averaged equation for inviscid disperse two-phase flow. J. Fluid Mech. 267, 185-219.

Zuber, N. \& Findlay, J. A. 1965 Average volumetric concentration in two-phase flow systems. Trans. ASME: J. Heat Transfer 87, 453-468. 